\title{
Atomic-scale structure of dislocations revealed by scanning tunneling microscopy and molecular dynamics
}

Christiansen, Jesper; Morgenstern, K.; Schiøtz, Jakob; Jacobsen, Karsten Wedel; Braun, K.F.; Rieder, K.H.; Laegsgaard, E.; Besenbacher, Flemming

Published in:

Physical Review Letters

Link to article, DOI:

10.1103/PhysRevLett.88.206106

Publication date:

2002

Document Version

Publisher's PDF, also known as Version of record

Link back to DTU Orbit

Citation (APA):

Christiansen, J., Morgenstern, K., Schiøtz, J., Jacobsen, K. W., Braun, K. F., Rieder, K. H., Laegsgaard, E., \& Besenbacher, F. (2002). Atomic-scale structure of dislocations revealed by scanning tunneling microscopy and molecular dynamics. Physical Review Letters, 88(20), 206106. https://doi.org/10.1103/PhysRevLett.88.206106

\section{General rights}

Copyright and moral rights for the publications made accessible in the public portal are retained by the authors and/or other copyright owners and it is a condition of accessing publications that users recognise and abide by the legal requirements associated with these rights.

- Users may download and print one copy of any publication from the public portal for the purpose of private study or research.

- You may not further distribute the material or use it for any profit-making activity or commercial gain

- You may freely distribute the URL identifying the publication in the public portal 


\title{
Atomic-Scale Structure of Dislocations Revealed by Scanning Tunneling Microscopy and Molecular Dynamics
}

\author{
J. Christiansen, ${ }^{1,2}$ K. Morgenstern, ${ }^{3,4, *}$ J. Schiøtz, ${ }^{1}$ K. W. Jacobsen, ${ }^{1}$ K.-F. Braun, ${ }^{3}$ K.-H. Rieder, ${ }^{3}$ \\ E. Lægsgaard, ${ }^{4}$ and F. Besenbacher ${ }^{4}$ \\ ${ }^{1}$ CAMP and Department of Physics, Technical University of Denmark, DK-2800 Lyngby, Denmark \\ ${ }^{2}$ Materials Research Department, Risø National Laboratory, DK-4000 Roskilde, Denmark \\ ${ }^{3}$ Institut für Experimentalphysik, FB Physik, Freie Universität Berlin, Arnimallee 14, D-14195 Berlin, Germany \\ ${ }^{4}$ CAMP and Department of Physics and Astronomy, University of Aarhus, DK-8000 Aarhus C, Denmark
}

(Received 12 October 2001; published 6 May 2002)

\begin{abstract}
The intersection between dislocations and a $\mathrm{Ag}(111)$ surface has been studied using an interplay of scanning tunneling microscopy (STM) and molecular dynamics. Whereas the STM provides atomically resolved information about the surface structure and Burgers vectors of the dislocations, the simulations can be used to determine dislocation structure and orientation in the near-surface region. In a similar way, the subsurface structure of other extended defects can be studied. The simulations show dislocations to reorient the partials in the surface region leading to an increased splitting width at the surface, in agreement with the STM observations. Implications for surface-induced cross slip are discussed.
\end{abstract}

DOI: $10.1103 /$ PhysRevLett.88.206106

Dislocations at surfaces play a major role in many areas of materials science. Dislocations intersecting surfaces are, for example, very important for the control of growth and solidification processes. Steps terminating at screw dislocations are ideal for continued crystal growth [1], and dislocations can strongly modify the surface stress important for atomic mobility and island nucleation in growth from the gas phase [2]. Dislocation-surface interactions also play a crucial role in determining the fracture toughness of a material since the emission and absorption of dislocations at a crack tip control the possible blunting of the tip [3].

However, the atomistic understanding of the behavior of dislocations at surfaces is still very scarce [4-9]. Bulk dislocations have in the past been studied extensively with electron microscopy (EM) and, in some cases, it has been possible to obtain atomic-scale information by imaging columns of atoms along a straight bulk dislocation [10]. It is not possible to obtain similar resolution with EM for dislocations at surfaces. Scanning tunneling microscopy (STM), on the other hand, is the technique of choice to reveal atomic-scale surface structures such as surface dislocations, but STM is blind with respect to the region beneath the topmost layer.

In this Letter, we show how STM observations of a dislocation intersecting a surface can be combined with atomistic simulations to provide detailed atomistic information about the structure of the dislocation in the near-surface region. The good agreement between experimentally observed and simulated surface structures gives credibility both to the interpretation of the surface structures as signatures of dislocations and to the calculated structures below the surface. The same principles can be used to study the interactions between surfaces and other extended defects such as grain boundaries.
PACS numbers: 68.35.Bs, 61.72.Bb, 61.72.Ff, 68.37.Ef
Consider a bulk dislocation that intersects a surface. If the total Burgers vector has a nonzero component along the surface normal, a surface step will end at the dislocation. If the dislocation is split into partial dislocations, a step may be seen between the partial dislocations, even if the total Burgers vector is parallel to the surface. From an atomic resolved STM image, the in-plane component of the Burgers vector can also be determined, allowing a direct determination of the Burgers vectors of the individual partial dislocations.

The STM cannot, however, determine the edge or screw character of the dislocations as it gives no information about the line vector of the dislocation. Information about the line vector and the subsurface structure can be obtained from an interplay with molecular dynamics simulations.

The STM measurements were performed on a single crystal $\operatorname{Ag}(111)$ surface in two ultrahigh vacuum systems equipped with standard facilities for sample preparation and characterization. One chamber houses a fast-scanning, variable-temperature STM [11], the other one a lowtemperature STM [12]. The clean $\operatorname{Ag}(111)$ surface was prepared by several sputtering-annealing cycles.

Several kinds of dislocations were found in the studied samples. In a number of cases, a surface step ends at a dislocation (see Fig. 1). Despite a significant amount of surface diffusion, the structure of the dislocation itself is very stable. Up to a step height of about $0.13 \mathrm{~nm}$, i.e., around $2 / 3$ of the full step height, the positions of the atoms do not change within half an hour. Above this step height, the step shows the frizziness expected for $\operatorname{Ag}(111)$ at this temperature, as the step is moving while the STM data are collected $[13,14]$. This inhibits the determination of the step profile with the same precision as on the lower parts of the step. 


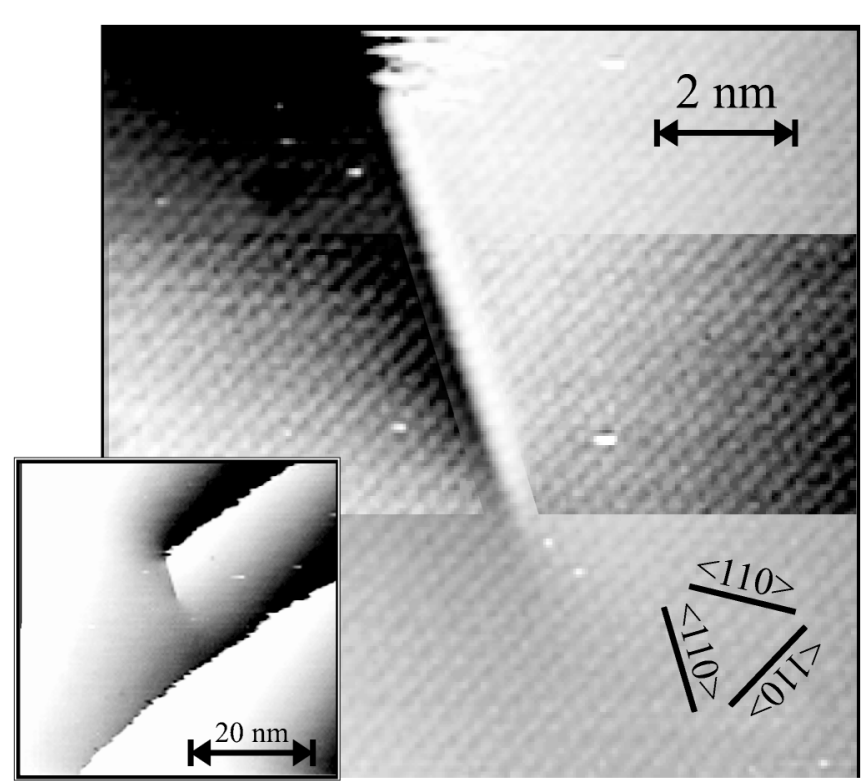

FIG. 1. Atomic resolution image of the intersection of a bulk dislocation with the $\operatorname{Ag}(111)$ surface and its surroundings (inset). The Burgers vector is $\frac{1}{2}\langle 110\rangle$. For better visibility, the contrast is enhanced on a broad stripe in the middle of the image to both the left side and the right side of the step, thus no tip change occurs. The horizontal shift in the atomic positions on the two sides of the extended dislocation is due to the in-surface component of the Burgers vectors of the partial dislocations. The STM parameters were $U=-0.22 \mathrm{~V}, I=1.4 \mathrm{nA}$, and $T=330 \mathrm{~K}$.

In rare cases, dislocations with a Burgers vector in the surface plane were observed, and in these cases only a minor perturbation of the surface is seen. In a few cases, some features which we interpret as Lomer-Cottrell locks (sessile dislocations with edge character [1]) were seen.

The simulations were performed using a previously determined effective medium theory potential $[15,16]$ for Ag. The potential has been fitted to the elastic constants, the vacancy formation energy, and the intrinsic stacking fault energy in accordance with experimental and ab initio data [17]. The simulations have been done at $0 \mathrm{~K}$ by minimizing the energy of the system, and at $300 \mathrm{~K}$ using Langevin dynamics with a time step of $5 \mathrm{fs}$ [18]. All simulation cells are rectangular with (111) surfaces at the top and the bottom of the cells, with free boundary conditions on all surfaces. It is not possible to have periodic boundary conditions in the directions perpendicular to the surface due to the net Burgers vector in the simulation cell. Care was taken to ensure that the systems were large enough to prevent unwanted interactions with the other surfaces of the system.

Figure 2 shows two calculated equilibrium configurations of a dislocation with a Burgers vector of $\boldsymbol{b}=\frac{1}{2}[110]$. Figure 2(a) corresponds to the lowest energy. The dislocation can dissociate according to one of the reactions,

$$
\frac{1}{2}[110] \rightarrow \frac{1}{6}[21 \overline{1}]+\frac{1}{6}[121] \quad \text { dissociating on }(1 \overline{1} 1),
$$
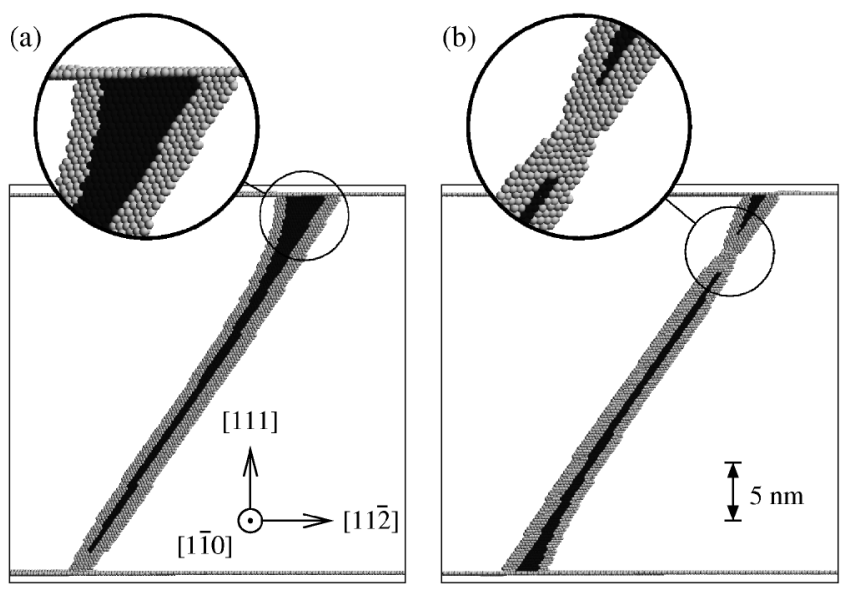

FIG. 2. Two configurations of a dislocation with $\boldsymbol{b}=\frac{1}{2}[110]$, corresponding to two different local minima of the energy. The simulated system contains approximately $2 \times 10^{6}$ atoms. The atoms in local fcc order and the atoms on the surfaces perpendicular to the (111) surfaces of the simulation cell have been removed, corresponding to showing $1 \%$ of the atoms. The atoms in local hcp order are dark while the atoms in the dislocation cores are grey. Configuration (a) has the lowest energy. Configuration (b) has a constriction where the slip plane of the dislocation changes, and this is shown in the blowup. Notice how the separation between the partials changes near the surfaces.

$$
\frac{1}{2}[110] \rightarrow \frac{1}{6}[12 \overline{1}]+\frac{1}{6}[211] \quad \text { dissociating on }(\overline{1} 11) .
$$

The simulation in Fig. 2(a) was set up as two partials according to reaction (1), with the partials initially separated by $2 \mathrm{~nm}$ at the surfaces, and the configuration was then allowed to relax. The equilibrium configuration has a line vector of [110] and is thus a screw dislocation, although this does not give the shortest dislocation length. The dislocation of Fig. 2(a) was initially set up with this line vector, but dislocations with other line vectors were seen to rotate to this orientation, starting at the surfaces.

Linear elasticity theory predicts that a screw dislocation has lower energy than an edge dislocation and that the elastic repulsion between two screw dislocations at right angles vanishes [1]. The elastic energy is thus significantly lowered if the partials rotate away from the bulk orientation to obtain a more screwlike character. This process is possible only because the surfaces break translation symmetry along the dislocation line, resulting in the changes seen near the surfaces in Fig. 2(a). Similar effects have been seen in simulations of a screw dislocation intersecting a $\mathrm{Cu}(110)$ surface [19].

The surface imprint of the dislocation at the upper surface in Fig. 2(a), where the distance between the partials is increased, corresponds well with the observed structure in Fig. 1. The profile of the surface step ending at the dislocation is shown in Fig. 3 for both the experiment and the simulation, and the splitting width as well as the widths of the individual partials agree well. 


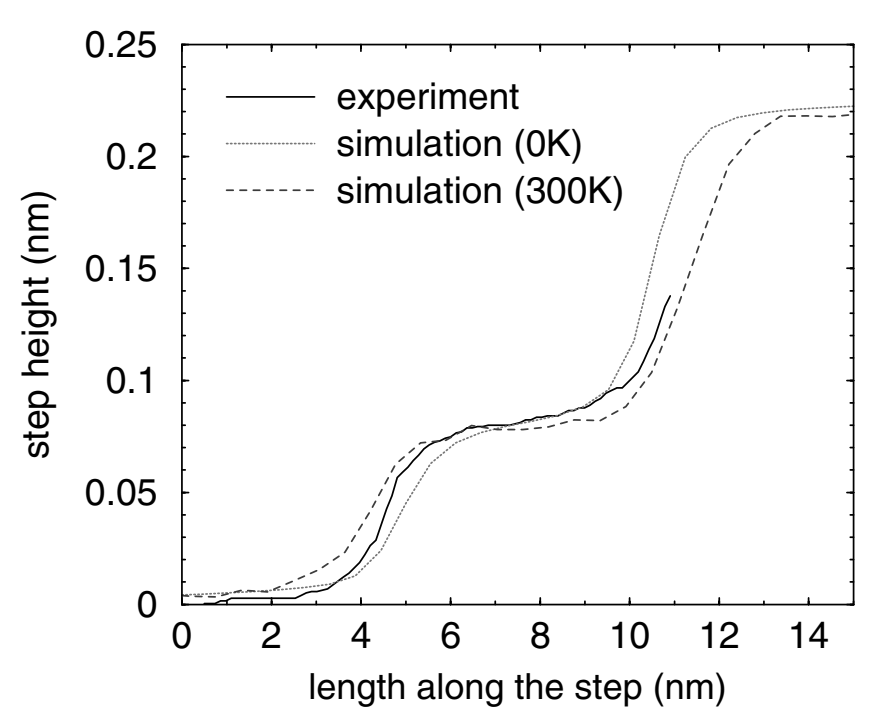

FIG. 3. Step profiles extracted from the STM image in Fig. 1 (solid line), from the simulation in Fig. 2(a) $(T=0 \mathrm{~K}$, dotted line), and from a similar simulation at $T=300 \mathrm{~K}$ (dashed line).

The direction of the surface step is not determined by the dislocation, and it may change if surface diffusion adds or removes atoms at the step [20]. In the simulations, the surface step was placed in the same way as in Fig. 1, i.e., adjacent to the partial with the largest out-of-surface component of the Burgers vector. If an extra half layer of atoms was added to the surface, the step would be in the opposite direction, and we would see a high step followed by a low step in Fig. 3.

Another subsurface structure is possible for the dislocation, that of Fig. 2(b), obtained when the energy of the perfect (undissociated) screw dislocation is minimized. In this case, dissociation begins at the surfaces according to reaction (1) at the top surface and reaction (2) at the bottom surface, in both cases creating partial dislocations rotated towards screw character. The screwlike constriction formed in Fig. 2(b) has a low energy. In $\mathrm{Cu}$, such a constriction has been shown to have negative energy compared to the straight dislocation [19].

The energy difference between the two configurations in Fig. 2 is $4.5 \mathrm{eV}$, favoring the unconstricted configuration in Fig. 2(a). The surface imprint of the constricted dislocation in Fig. 2(b) is not seen in the STM. Notice, however, that TEM studies of dislocations in thin foils of $\mathrm{Cu} 10$ at. \% $\mathrm{Al}[21,22]$ revealed both structures in Fig. 2. Also, Fig. 2(a) indicates that a second much lower splitting width of about $1 \mathrm{~nm}$ with no discernible plateau between the partials should be observed experimentally, corresponding to the configuration at the lower surface of Fig. 2(a). If the dislocation had been dissociated on the

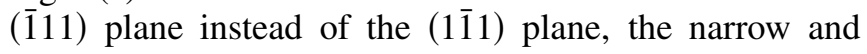
wide ends would have been swapped, and both configurations are therefore expected at the same surface. We do, however, not observe the narrow configuration, except for dislocations with the Burgers vector in the surface plane (see Fig. 4), where both configurations are seen.

The altered dislocation structure near the surface may influence cross slip rates of screw dislocations. Cross slip is the process by which a screw dislocation changes glide plane and is an important process in metals in the late stages of work hardening [23]. As the cross slip process requires two partial dislocations to first recombine into a perfect dislocation, the rate depends critically on the separation of the partials. The dislocation in Fig. 2(a) will have a much increased probability of cross slip near the bottom surface, but not near the top. The dislocation in Fig. 2(b) has effectively already begun the cross slip process, which can proceed by moving the constriction down along the dislocation.

Figures 4(a) and 4(b) show dislocations with Burgers vectors in the surface plane at two different temperatures.
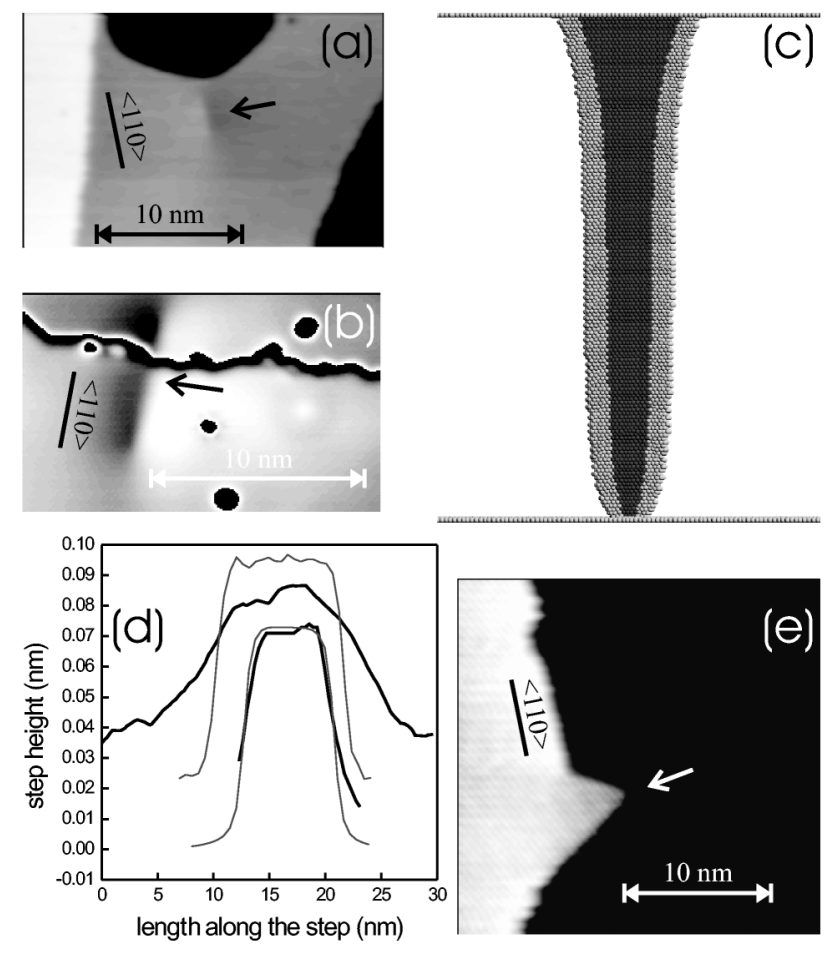

FIG. 4. Observations and simulations of dislocations and observation of a Lomer-Cottrell lock. In (a) and (b) we show STM images of dislocations with Burgers vectors in the surface plane, emerging at the arrows. The temperature was $T=318$ and $7.5 \mathrm{~K}$, respectively. The semicircle in (a) is a vacancy island. The dislocation in (b) crosses a surface step. The image contrast has been chosen such that grey scales on the two terraces are identical. The simulated subsurface structure of such dislocations at $T=0 \mathrm{~K}$ is shown in (c). The step profiles of the dislocations [thick lines, (a) at the top, (b) at the bottom] and the simulated step profiles at $T=300$ and $0 \mathrm{~K}$ (thin lines, top and bottom, respectively) are shown in (d). The upper curves are shifted upwards, and the low-temperature experimental curve has been shifted to lie on the simulated curve, since the total height could not be determined in the experiment. In (e) we show a structure consistent with a Lomer-Cottrell lock pinning a surface step. 
No surface step ends at the dislocation, but it is nevertheless visible as it splits into partials that each have a Burgers vector with a component perpendicular to the surface. The dislocation in Fig. 4(b) crosses a step, but this is of minor consequence since the elastic field of a step is small.

In Fig. 4(d), we show step profiles along the dislocations in Figs. 4(a) and 4(b) as well as from simulations at $T=300$ and $0 \mathrm{~K}$. The dislocation dissociates according to the reaction $\frac{1}{2}[1 \overline{1} 0] \rightarrow \frac{1}{6}[1 \overline{2} \overline{1}]+\frac{1}{6}[2 \overline{1} 1]$. Even though the height of the experimental curve could not be determined, good agreement is found between simulations and experiment. The agreement is less good at the higher temperatures. Part of the experimental broadening is due to lower resolution of the image itself and especially of the tip. While the step edge in Fig. 4(b) has an apparent width of $1 \mathrm{~nm}$ (consistent with a perfect tip), the step edge in Fig. 4(a) has an apparent width of $2 \mathrm{~nm}$.

The splitting width of the dislocations, i.e., the distance between the centers of the two partial dislocations, can easily be obtained from the step profiles. For the dislocation in Fig. 1, we obtain a splitting width of $6.4 \mathrm{~nm}$, to be compared with 5.5 and $7.2 \mathrm{~nm}$ in the simulations at 0 and $300 \mathrm{~K}$, respectively. The experimental data is insufficient to reliably extract splitting width for the dislocations in Fig. 4; the splitting widths in the simulations are 8.1 and $11 \mathrm{~nm}$ at 0 and $300 \mathrm{~K}$, respectively. All these values are significantly higher than the bulk splitting widths, measured to be $d_{\mathrm{s}} \simeq 2.1 \mathrm{~nm}$ for screw dislocations in silver, and $d_{\mathrm{e}} \simeq 8.5 \mathrm{~nm}$ for edge dislocations [24]. In our simulations, the splitting widths far from the surfaces are $d_{\mathrm{s}} \simeq 1.5$ and $6.6 \mathrm{~nm}$, in reasonable agreement with the experimental values.

Two mobile dislocations may collide and form a LomerCottrell (LC) lock which is a sessile edge dislocation that splits on two different planes. This can, e.g., occur according to the reaction

$$
\begin{aligned}
\frac{1}{2}[\overline{1} 01]+\frac{1}{2}[01 \overline{1}] & \rightarrow \frac{1}{2}[\overline{1} 10] \\
& \rightarrow \frac{1}{6}[\overline{1} 1 \overline{2}]+\frac{1}{6}[\overline{1} 10]+\frac{1}{6}[\overline{1} 12],
\end{aligned}
$$

with the line vector along [110]. A surface structure consistent with such a dislocation pinning a surface step is seen in Fig. 4(e). The angle of the sharp v-shape is consistent with the angle between the splitting planes of a LC lock. The step height changes by one third at the two Shockley partials, since they have a component of the Burgers vector perpendicular to the surface. Although there is complete symmetry between the two Shockley partials, the lowest energy configuration spontaneously breaks this symmetry. Linear elasticity theory predicts a ratio between the splitting distances of 3.8:1 [25]. A lower ratio is clearly seen in Fig. 4(e), probably from the influence of the surface. Unfortunately, the large splitting distances make an atomistic simulation with a realistic potential prohibitively expensive.
In summary, we have recorded STM images of dislocations intersecting the $\operatorname{Ag}(111)$ surface and measured step profiles of the resulting surface steps. These STM experiments have been compared to atomic-scale simulations with good agreement, even on the quantitative level. We find that this combination of STM and atomic-scale simulations provides a powerful method for studying the surface-induced structural changes of crystal defects.

This work was supported by the Danish Research Councils through Grant No. 5020-00-0012. The Center for Atomic-Scale Materials Physics (CAMP) is sponsored by the Danish National Research Foundation.

*Corresponding author.

Electronic address: karina.morgenstern@ @ physik.fu-berlin. de

[1] J. P. Hirth and J. Lothe, Theory of Dislocations (McGrawHill, New York, 1968).

[2] H. Brune, Surf. Sci. Rep. 31, 121 (1998).

[3] A. E. Carlsson and R. Thomson, Solid State Phys. 51, 233 (1998).

[4] J. de la Figuera et al., Phys. Rev. B 58, 1169 (1998).

[5] O. Rodríguez de la Fuente, M. A. González, and J. M. Rojo, Phys. Rev. B 63, 085420 (2001).

[6] L. D. Marks, Phys. Rev. Lett. 51, 1000 (1983).

[7] G. Cox et al., Phys. Rev. Lett. 64, 2402 (1990).

[8] A. Samsavar et al., Phys. Rev. Lett. 65, 1607 (1990).

[9] J. Wolf and H. Ibach, Appl. Phys. A 52, 218 (1991).

[10] J. C. H. Spence, Mater. Sci. Eng. R 26, 1 (1999).

[11] F. Besenbacher, Rep. Prog. Phys. 59, 1737 (1996).

[12] G. Meyer, Rev. Sci. Instrum. 67, 2960 (1996).

[13] K. Morgenstern, G. Rosenfeld, B. Poelsema, and G. Comsa, Phys. Rev. Lett. 74, 2058 (1995).

[14] M. Poensgen, J. F. Wolf, J. Frohn, M. Giesen, and H. Ibach, Surf. Sci. 274, 430 (1992).

[15] K. W. Jacobsen, J. K. Nørskov, and M. J. Puska, Phys. Rev. B 35, 7423 (1987).

[16] K. W. Jacobsen, P. Stoltze, and J. K. Nørskov, Surf. Sci. 366, 394 (1996).

[17] T. Rasmussen, Phys. Rev. B 62, 12664 (2000).

[18] M. P. Allen and D. J. Tildesley, Computer Simulation of Liquids (Clarendon, Oxford, 1987).

[19] T. Rasmussen, K. W. Jacobsen, T. Leffers, and O. B. Pedersen, Phys. Rev. B 56, 2977 (1997).

[20] K. Morgenstern et al. (to be published).

[21] P. M. Hazzledine, H. P. Karnthaler, and E. Wintner, Philos. Mag. 32, 81 (1975).

[22] E. Wintner, H. P. Karnthaler, and P. M. Hazzledine, in Proceedings of the 4th International Conference on the Strength of Metals and Alloys (Laboratoire de Physique du Solide, Nancy, 1976), Vol. 2, p. 927.

[23] A. Seeger, R. Berner, and H. Wolf, Z. Phys. 155, 247 (1959).

[24] D. J. H. Cockayne, M. L. Jenkins, and I. L. F. Ray, Philos. Mag. 24, 1383 (1971).

[25] J. Bonneville and J. Douin, Philos. Mag. Lett. 62, 247 (1990). 